\title{
COMPARAÇÃO DAS ESTRUTURAS DE CONTINUIDADE ESPACIAL EM QUATRO FORMAÇÕES FLORESTAIS DO ESTADO DE SÃO PAULO
}

\author{
Melissa Oda-Souza ${ }^{1}$, João Luís Ferreira Batista ${ }^{2}$, Paulo Justiniano Ribeiro $\mathrm{Jr}^{3}$, Ricardo Ribeiro Rodrigues ${ }^{4}$ \\ ${ }^{1}$ Eng $^{\mathrm{a}}$. Agrônoma, Dr., ESALQ/USP, Piracicaba, SP, Brasil - melissa.oda @ gmail.com \\ ${ }^{2}$ Eng. Florestal, Ph.D., Depto. de Ciências Florestais, ESALQ/USP, Piracicaba, SP, Brasil - parsival@usp.br \\ ${ }^{3}$ Eng. Agrônomo, Ph.D., Depto. de Estatística, UFPR, Curitiba, PR, Brasil - paulojus@ufpr.br \\ ${ }^{4}$ Biológo, Dr., Depto. de Ciências Biológicas, ESALQ/USP, Piracicaba, SP, Brasil - rrr@esalq.usp.br \\ Recebido para publicação: 02/07/2009 - Aceito para publicação: 21/02/2010
}

\begin{abstract}
Resumo
A teoria clássica de amostragem aplicada a estudos ecológicos geralmente tem o objetivo de realizar inferência sobre os parâmetros de comunidades e populações. No entanto, muitas vezes o interesse é também o reconhecimento do padrão espacial das comunidades. Neste trabalho foram ajustados modelos que permitem comparar as estruturas espaciais de quatro parcelas permanentes instaladas em diferentes formações florestais do estado de São Paulo. Nessas quatro parcelas foram obtidos dados de área basal, biomassa e densidade para sub-parcelas de $20 \times 20$ m considerando todas as árvores da parcela, árvores localizadas no estrato inferior e médio (estrato sub-bosque) e árvores localizadas no estrato dossel e emergente (estrato dossel). O ajuste e a comparação das estruturas de continuidade espacial das quatro formações florestais foram realizadas por meio de modelagem geoestatística e a seleção de modelos pelo critério de informação de Akaike. Verificou-se que as estruturas de continuidade espacial são distintas entre estratos nas quatro formações, sendo mais visíveis no subbosque. Considerando as formações, verificou-se que as estruturas espaciais são diferentes, mais notáveis para a Savana e Ombrófila.
\end{abstract}

Palavras-chave: Floresta tropical; estratos; modelagem geoestatística; máxima verossimilhança; critério de informação de Akaike.

\begin{abstract}
Spatial continuity structures comparison in four forest formation in the State of São Paulo, Brazil. The classical sampling theory applied to ecological studies generally aim to perform inference on the parameters of communities and populations. However, many times the interest is also the recognition of the community's spatial pattern. In this study models were adjusted for comparing the spatial structures of four forest formations. Four permanent plots located in different forest formations in São Paulo State were used. Data of basal area, biomass and density were obtained from sub-plots of $20 \times$ $20 \mathrm{~m}$ considering all the trees in the plot, trees located in the lower and intermediate strata (understory stratum) and trees located in the canopy and emerging strata (canopy layer). The adjustment and comparison of the structures of spatial continuity of the four forest formations were performed by means of a geostatistic model, and the selection of models by the Akaike information criterion. It was found that the structures of spatial continuity were distinct between the four forest formations strata, being most pronounced in the understory. Considering the forest formation it was found that the spatial structures were different, more pronounced for the Savanna and Dense Rain Forest.

Keywords: Tropical forest; strata; geostatistical modeling; maximum likelihood; Akaike information criterion.
\end{abstract}

\section{INTRODUÇÃO}

A maior parte dos fenômenos ecológicos variam no espaço e tempo, por exemplo: densidades populacionais variam de ano para ano, e raramente são idênticas de um local para outro; as taxas de dispersão variam de acordo com a densidade populacional e, portanto, são diferentes de um ano para o outro e de uma população para outra; e as condições climáticas variam tanto anualmente quanto 
localmente (KOENIG, 1999).

Nesse sentido, essas florestas devem ser monitoradas periodicamente por métodos que permitam quantificar a diversidade biológica, a fim de acumular conhecimento e gerar informações precisas sobre diferentes processos ecológicos (RODRIGUES, 2002).

Há muito tempo é reconhecido que procedimentos de amostragem desempenham um papel importante no estudo de populações e comunidades em ecologia, sendo utilizados procedimentos da teoria clássica de amostragem (COCHRAN, 1977). Embora compreendendo que muito desses princípios são fundamentais (por exemplo, aleatorização), também é importante reconhecer que os objetivos de um estudo ecológico podem diferir daqueles considerados pela teoria clássica. Especificamente, a teoria clássica de amostragem é amplamente envolvida na inferência sobre os parâmetros de comunidades e populações, mas, em investigações ecológicas, o interesse principal é usualmente o reconhecimento do padrão espacial das comunidades (KENKEL et al., 1989).

Estudos ecológicos apontam que, se não todas, a maioria das comunidades são espacialmente estruturadas e que os métodos de amostragem devem ser robustos, para detectar diferentes níveis de autocorrelação espacial (GOSLEE, 2006).

Uma possível forma de modelar esse padrão espacial incorporando a estrutura de continuidade espacial é pela abordagem de modelagem geoestatística que associa o grau de dependência espacial a medidas de distância e direção entre os pontos amostrados (VIOLA, 2007). Além de incorporar a estrutura de continuidade espacial, tais modelos permitem predizer valores em pontos não amostrados, possibilitando a construção de mapas que descrevem a distribuição espacial de variáveis de interesse.

Considerando que as formações florestais podem apresentar diferentes estruturas de continuidade espacial, foram ajustados modelos visando comparar as estruturas espaciais em quatro formações florestais distintas do estado de São Paulo.

\section{MATERIAL E MÉTODOS}

\section{Áreas de estudo}

Os dados utilizados nesse trabalho são provenientes do Projeto "Diversidade, dinâmica e conservação em florestas do estado de São Paulo: 40 ha de parcelas permanentes", do Programa Biota, da FAPESP (RODRIGUES, 2002; 2005). Foram instaladas parcelas permanentes em quatro Unidades de Conservação do estado de São Paulo (Figura 1A), que representam as principais formações florestais do estado: Floresta de Restinga (Parque Estadual Ilha do Cardoso), Floresta Ombrófila Densa Montana (Parque Estadual de Carlos Botelho), Floresta Estacional Semidecidual (Estação Ecológica dos Caetetus) e Cerrado lato sensu ou Savana Florestada (Estação Ecológica de Assis). Em cada Unidade de Conservação, foi alocada uma parcela permanente de $320 \times 320 \mathrm{~m}$, totalizando 10,24 ha, subdividida em 256 subparcelas contíguas de $20 \times 20 \mathrm{~m}\left(400 \mathrm{~m}^{2}\right)$ (Figura 1B). Todos os indivíduos arbóreos no interior das parcelas com diâmetro (d - diâmetro à altura do peito) maior ou igual a $5 \mathrm{~cm}$ foram mapeados, georreferenciados, medidos e identificados. Com essas informações, foram construídos mapas de todas as árvores das parcelas permanentes, com suas coordenadas.

Nas quatro parcelas permanentes, obtiveram-se dados de área basal $\left(\mathrm{m}^{2} \cdot \mathrm{ha}^{-1}\right)$, biomassa $\left(\mathrm{Mg} \cdot \mathrm{ha}^{-1}\right)$ e densidade $\left(\mathrm{ha}^{-1}\right)$ para as subparcelas de $20 \times 20 \mathrm{~m}$, considerando-se todas as árvores da parcela, árvores localizadas no estrato inferior e médio (estrato sub-bosque) e árvores localizadas no estrato dossel e emergente (estrato dossel), conforme a tabela 1.

Tabela 1. Número de árvores no estrato total, sub-bosque e dossel nas quatro formações florestais. Table 1. Number of trees in stratum total, understory and canopy in the four forest formations.

\begin{tabular}{lccc}
\hline Formações & Total & Sub-bosque & Dossel \\
\hline Savana Florestada & 21.861 & $18.623(5<\mathrm{d}<15 \mathrm{~cm})$ & $3.238(\mathrm{~d} \geq 15 \mathrm{~cm})$ \\
Floresta Estacional & 11.904 & $10.185(5<\mathrm{d}<20 \mathrm{~cm})$ & $1.719(\mathrm{~d} \geq 20 \mathrm{~cm})$ \\
Floresta Ombrófila & 11.387 & $8.810(5<\mathrm{d}<15 \mathrm{~cm})$ & $2.577(\mathrm{~d} \geq 15 \mathrm{~cm})$ \\
Floresta de Restinga & 15.425 & $11.518(5<\mathrm{d}<15 \mathrm{~cm})$ & $3.907(\mathrm{~d} \geq 15 \mathrm{~cm})$ \\
\hline
\end{tabular}

A biomassa da parcela foi obtida totalizando a biomassa total acima do solo das árvores individuais, utilizando-se os modelos apresentados na tabela 2 . 

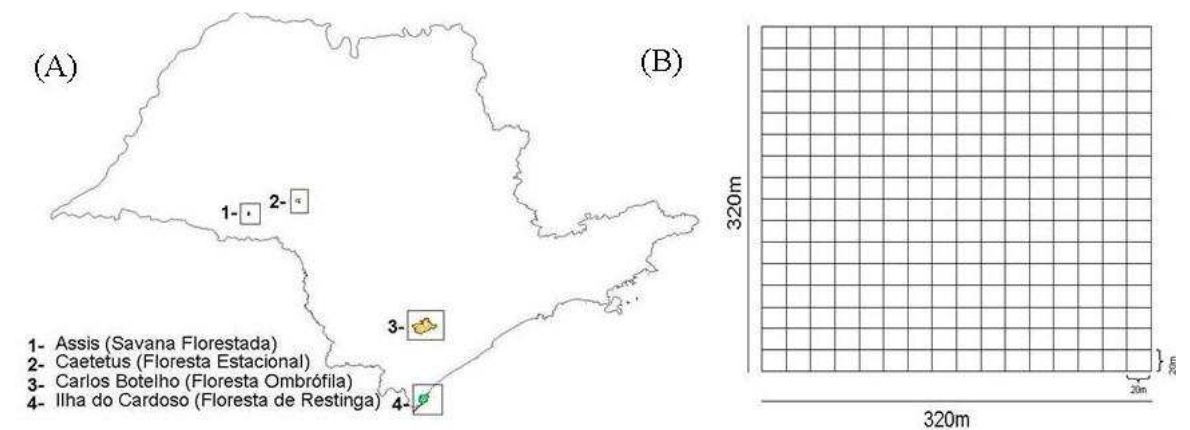

Figura 1. (A) Localização das quatro Unidades de Conservação no estado de São Paulo; (B) Parcela permanente subdividida em 256 subparcelas contíguas de $20 \times 20 \mathrm{~m}\left(400 \mathrm{~m}^{2}\right)$.

Figure 1. (A) Location of the four Conservation Units of the state of São Paulo; (B) Permanent plot subdivided into 256 contiguous subplots of $20 \times 20 \mathrm{~m}\left(400 \mathrm{~m}^{2}\right)$.

Tabela 2. Modelos para estimativas das biomassas das árvores individuais.

Table 2. Models to estimate the biomass of individual trees.

\begin{tabular}{lcc}
\hline Formações florestais & Modelo & Fonte \\
\hline Savana Florestada e Restinga & $\mathrm{b}=\exp (-1,79532)(\mathrm{d})^{2,29744}$ & Batista, 2008 (dados não publicados) $^{1}$ \\
Estacional & $\mathrm{b}=\exp (-9,36745+2,47442(\log (\mathrm{d})))$ & Mello,2008 (dados não publicados) \\
Ombrófila & $\mathrm{b}=\exp (-6.80067+3,77738(\log (\mathrm{d})))$ & Burger, 2005 $^{2}$ \\
\hline
\end{tabular}

b: biomassa $\left(\mathrm{Mg} \cdot h a^{-1}\right)$; d: diâmetro à altura do peito $(\mathrm{cm})$.

\section{Modelos utilizados na comparação das estruturas espaciais}

Para a comparação das estruturas de continuidade espacial das quatro formações florestais nos diferentes estratos, foram adotados cinco modelos (Tabela 3). A primeira alternativa considerada foi a de modelar as quatro formações florestais com a mesma estrutura espacial (M1); o segundo modelo (M2) considerou as formações com estruturas espaciais diferentes; os modelos M3 e M5 tiveram como base a semelhança do padrão espacial das árvores das florestas Savana e Restinga e o padrão espacial mais pronunciado das árvores da floresta Ombrófila, respectivamente, descrito por Capretz (2004); o modelo M4 considerou a proximidade entre as florestas de Savana e Estacional.

Tabela 3. Modelos propostos para a comparação das estruturas espaciais das quatro formações florestais (S- Savana, E- Estacional, O- Ombrófila e R- Restinga).

Table 3. Proposed models for the comparison of spatial structures in the four forest formations (SSavanna, E- Seasonal Semideciduous, O- Dense Rain, R- Restinga).

\begin{tabular}{lll}
\hline & Modelos & Parâmetros $\left(\mu_{k} ; \sigma_{k}^{2} ; \tau_{k}^{2} ; \varphi_{k}\right)$ \\
\hline M1 & $\mathrm{S}=\mathrm{E}=\mathrm{O}=\mathrm{R}$ & $\mathrm{k}=0$ \\
M2 & $\mathrm{S} \neq \mathrm{E} \neq \mathrm{O} \neq \mathrm{R}$ & $\mathrm{k}=1,2,3,4(1=\mathrm{S} ; 2=\mathrm{E}, 3=\mathrm{O}, 4=\mathrm{R})$ \\
M3 & $(\mathrm{S}=\mathrm{R}) \neq \mathrm{E} \neq \mathrm{O}$ & $\mathrm{k}=1,2,3(1=\mathrm{S} ; \mathrm{R}, 2=\mathrm{E}, 3=\mathrm{O})$ \\
M4 & $(\mathrm{S}=\mathrm{E}) \neq \mathrm{O} \neq \mathrm{R}$ & $\mathrm{k}=1,2,3(1=\mathrm{S} ; \mathrm{E}, 2=0,3=\mathrm{R})$ \\
M5 & $(\mathrm{S}=\mathrm{E}=\mathrm{R}) \neq \mathrm{O}$ & $\mathrm{k}=1,2(1=\mathrm{S} ; \mathrm{E} ; \mathrm{R} ; 2=\mathrm{O})$ \\
\hline
\end{tabular}

\section{Modelagem da estrutura espacial}

No contexto de delineamento de amostragem, $\mathrm{x}_{\mathrm{i}}$ representa a posição da unidade amostral e $\mathrm{Y}_{\mathrm{i}}$ as variáveis de medição, como área basal, biomassa e densidade. $\mathrm{O}$ formato básico de dados geoestatísticos é $\left(x_{i}, y_{i}\right), i=1, \ldots, n$, em que $x_{i}$ identifica a posição espacial tipicamente em um plano bidimensional e $y_{i}$ é uma medição tomada na posição $\mathrm{x}_{\mathrm{i}}$. Uma característica da modelagem geoestatística é que a variável $\mathrm{Y}_{\mathrm{i}}$

${ }^{1}$ BATISTA, J. L. F. Escola Superior de Agricultura "Luiz de Queiroz", Universidade de São Paulo, Departamento de Ciências Florestais, 2008.

${ }^{2}$ MELLO, J. M. Universidade Federal de Lavras, Departamento de Ciências Florestais, 2008.

FLORESTA, Curitiba, PR, v. 40, n. 3, p. 515-522, jul./set. 2010. 
pode, em princípio, estar localizada em qualquer lugar numa região de estudo (DIGGLE; RIBEIRO JR., 2007). Sob a suposição de que os dados de área basal, biomassa e densidade $\mathrm{Y}=\mathrm{Y}_{1}, \ldots, \mathrm{Y}_{\mathrm{n}}$ seguem $\mathrm{o}$ modelo geoestatístico linear gaussiano exposto por Diggle et al. (1998) e Diggle; Ribeiro Jr. (2007), a dependência espacial é modelada através de um componente de erro aleatório e espacialmente correlacionado, de forma que $\mathrm{Y}_{\mathrm{i}}=\mu\left(\mathrm{x}_{\mathrm{i}}\right)+\mathrm{S}\left(\mathrm{x}_{\mathrm{i}}\right)+\varepsilon_{\mathrm{i}}, \mathrm{i}=1, \ldots, \mathrm{n}$, em que $\mathrm{Y}_{\mathrm{i}}$ é a variável de interesse, $\mathrm{x}_{\mathrm{i}}$ é a posição espacial da i-ésima parcela e $\mu\left(\mathrm{x}_{\mathrm{i}}\right)$ é determinada a partir de um modelo de regressão. $\mathrm{O}$ termo $\mathrm{S}\left(\mathrm{x}_{\mathrm{i}}\right)$ denota o valor na posição de um processo estacionário gaussiano, com média zero, variância $\sigma^{2} \mathrm{e}$ função de correlação $\rho(u ; \phi)$, com argumento dado pela distância u de separação entre observações e parametrizada por $\phi$, que descreve o grau de associação espacial. Decorre então que, para um conjunto finito de observações $S \sim \operatorname{NMV}\left(0, \sigma^{2} \mathrm{R}(\phi)\right)$, em que $\mathrm{R}(\phi)$ é a matriz de covariância $\mathrm{n} \times \mathrm{n}$ com $(\mathrm{i}, \mathrm{j})$-ésimo elemento, $\rho(u)$, sendo $u=\left\|x_{i}-x_{j}\right\|$ a distância euclidiana entre $x_{i}$ e $x_{j}$. O termo $\varepsilon_{i}$ denota variáveis aleatórias, independentes e normalmente distribuídas, com média zero e variância $\tau^{2}$. A função de correlação $\rho(\mathrm{u})$ adotada aqui e que define os valores na matriz de covariância foi a exponencial $\rho(\mathrm{u})=\exp \{-(\mathrm{u} / \phi)\}$, e o alcance prático é aproximadamente $3 \phi$. Seguindo a terminologia geoestatística usual (ISAAKS; SRIVASTAVA, 1989), $\tau^{2}$ é o efeito pepita (nugget), $\sigma^{2}$ é o patamar (sill) e a distância a partir da origem até o patamar é chamada de alcance prático (range), que denotamos aqui por $a$, que de forma geral é função do parâmetro $\phi$ e $a=3 \phi$ para a função de correlação exponencial. A razão entre efeito pepita $\left(\tau^{2}\right)$ com o sill total $\left(\tau^{2}+\sigma^{2}\right)$ é chamado de efeito pepita relativo $\left(\operatorname{Rel}_{\tau^{2}}\right)$, que representa a proporção do componente aleatório sobre a variância total. Para a transformação do modelo gaussiano, foi aplicada a família de transformação Box-Cox (BOX; COX, 1964), que é discutida no contexto de modelagem geoestatística por Christensen et al. (2001). A estimação dos parâmetros foi obtida por máxima verossimilhança (DIGGLE; RIBEIRO JR., 2007).

\section{Seleção dos modelos}

A avaliação do desempenho dos modelos foi realizada em duas etapas. Na primeira, avaliou-se a plausibilidade das diferentes funções de correlação nos modelos espaciais, e, na segunda, a plausibilidade dos modelos espaciais e não-espaciais. As duas etapas foram baseadas no critério de informação de Akaike (AIC). Akaike (1974) estabeleceu uma relação entre a informação ou distância de KullbackLeibler (KULLBACK; LEIBLER, 1951), que é uma medida de discrepância relativa entre dois modelos, o modelo verdadeiro e o modelo aproximado, e o máximo da função de log-verossimilhança para seleção de modelos. O AIC é definido por AIC $=-2 l+2 p$, em que $l$ é o logaritmo neperiano do máximo da função de verossimilhança e $p$ é o número de parâmetros do modelo considerado.

O valor de AIC não é interpretável individualmente para um único modelo. Uma alternativa proposta por Burnham; Anderson (2002) para avaliar a plausibilidade dos modelos é baseada nas diferenças dos $\operatorname{AIC}\left(\Delta_{\mathrm{i}}\right)$ e dada por $\Delta_{\mathrm{i}}=\mathrm{AIC}_{\mathrm{i}}-\mathrm{AIC}_{\mathrm{m}}$, em que $\mathrm{AIC}_{\mathrm{i}}$ é o valor de $\mathrm{AIC}$ para o $i$-ésimo modelo e $\mathrm{AIC}_{\mathrm{m}}$ é o valor de $\mathrm{AIC}_{\mathrm{m}}$ para o modelo com menor valor de AIC. Para valores de $\Delta_{\mathrm{i}}$ maiores do que dois, o modelo i é menos plausível que o modelo m. Os valores das diferenças dos $\operatorname{AIC}\left(\Delta_{\mathrm{i}}\right)$ são de fácil interpretação e permitem uma rápida comparação de modelos ordenados.

Especificamente para avaliar a plausibilidade dos modelos espaciais e não-espaciais, fixou-se o $\mathrm{AIC}_{\mathrm{i}}$ como o valor do AIC para o modelo não-espacial ( $\mathrm{AIC}_{\mathrm{nsp}}$ ) e o $\mathrm{AIC}_{\mathrm{m}}$ como o valor do $\mathrm{AIC}$ para o modelo espacial $\left(\mathrm{AIC}_{\mathrm{sp}}\right.$ ), e foi obtido por $\Delta_{\mathrm{sp}}=\mathrm{AIC}_{\mathrm{nsp}}-\mathrm{AIC}_{\mathrm{sp}}$. Para valores de $\Delta_{\mathrm{sp}}$ maiores do que dois, $\mathrm{o}$ modelo espacial é mais plausível que o modelo não-espacial.

Neste estudo, as análises foram realizadas utilizando-se o pacote geoR (RIBEIRO JR.; DIGGLE, 2001), do projeto R de computação estatística (R DEVELOPMENT CORE TEAM, 2008).

\section{RESULTADOS E DISCUSSÃO}

Na tabela 4 são apresentados os valores das diferenças dos AIC $\left(\Delta_{\mathrm{sp}}\right)$ e as estimativas dos parâmetros do modelo geoestatítico nos estratos total, sub-bosque e dossel, para as variáveis área basal, biomassa e densidade, nas quatro formações florestais estudadas.

Pode-se observar pelos valores das diferenças dos $\operatorname{AIC}\left(\Delta_{\mathrm{sp}}\right)$ que os modelos espaciais são mais plausíveis nas florestas de Savana, Ombrófila e de Restinga para os todos os estratos e variáveis. No entanto as estruturas espaciais das florestas Ombrófila e Savana são marcadamente diferentes, mais visíveis do que nas florestas Estacional e de Restinga. Os valores de alcance são maiores na Savana do que na Ombrófila, considerando todas as variáveis e estratos estudados. Esses resultados sugerem que a estrutura de continuidade espacial da Savana se apresenta em maior escala do que na Ombrófila. 
Tabela 4. Informação de Akaike (AIC) para modelo espacial $\left(\mathrm{AIC}_{\mathrm{sp}}\right)$ e não-espacial $\left(\mathrm{AIC}_{\mathrm{nsp}}\right)$, diferenças dos $\mathrm{AIC}$ $\left(\Delta_{\mathrm{sp}}\right)$ e estimativas dos parâmetros $\left(\sigma^{2}, \tau^{2}\right)$, efeito pepita relativo $\left(\operatorname{Rel}_{\tau^{2}}\right)$, alcance prático (a) do modelo geoestatístico nos estratos total (T), sub-bosque (SB) e dossel (D) nas quatro formações florestais.

Table 4. Akaike information criterion for spatial models $\left(\mathrm{AIC}_{\mathrm{sp}}\right)$ and non-spatial $\left(\mathrm{AIC}_{\mathrm{nsp}}\right)$, AIC differences $\left(\Delta_{\mathrm{sp}}\right)$ and estimates of the parameters $\left(\sigma^{2}, \tau^{2}\right)$, relative nugget $\left(\operatorname{Rel}_{\tau^{2}}\right)$, practical range (a) of the geostatistical model in stratum total (T), understory (SB) and canopy (D) in the four forest formations.

\begin{tabular}{|c|c|c|c|c|c|c|c|c|c|c|}
\hline \multirow{2}{*}{ Formação } & \multirow{2}{*}{ Variável } & \multirow{2}{*}{ Estratos } & \multicolumn{2}{|c|}{ AIC } & \multirow{2}{*}{$\Delta_{\mathrm{sp}}$} & \multicolumn{5}{|c|}{ Espacial } \\
\hline & & & $\mathbf{A I C}_{\mathrm{sp}}$ & $\mathbf{A I C}_{\text {nsp }}$ & & $\sigma^{2}$ & $\tau^{2}$ & $\sigma^{2}+\tau^{2}$ & $\operatorname{Rel}_{\tau^{2}}$ & $\mathbf{a}$ \\
\hline \multirow{9}{*}{ Savana } & \multirow{3}{*}{ A. basal } & $\mathrm{T}$ & 668,80 & 690,00 & 21,20 & 0,23 & 0,64 & 0,87 & 0,73 & 218,03 \\
\hline & & SB & 516,91 & 667,20 & 150,29 & 0,85 & 0,29 & 1,14 & 0,25 & 733,84 \\
\hline & & $\mathrm{D}$ & 816,49 & 839,59 & 23,10 & 0,56 & 1,03 & 1,59 & 0,65 & 158,99 \\
\hline & \multirow{3}{*}{ Biomassa } & $\mathrm{T}$ & 547,04 & 557,39 & 10,35 & 0,11 & 0,41 & 0,52 & 0,79 & 183,44 \\
\hline & & SB & 410,29 & 557,52 & 147,22 & 0,57 & 0,20 & 0,77 & 0,26 & 846,88 \\
\hline & & $\mathrm{D}$ & 776,28 & 797,27 & 20,99 & 0,42 & 0,95 & 1,37 & 0,70 & 222,78 \\
\hline & \multirow{3}{*}{ Densidade } & $\mathrm{T}$ & 1792,49 & 1929,74 & 137,25 & 108,98 & 32,58 & 141,56 & 0,23 & 345,14 \\
\hline & & SB & 1820,58 & 1975,30 & 154,71 & 141,91 & 26,49 & 168,40 & 0,16 & 269,60 \\
\hline & & $\mathrm{D}$ & 1607,82 & 1626,99 & 19,17 & 19,50 & 13,61 & 33,11 & 0,41 & 58,09 \\
\hline \multirow{9}{*}{ Estacional } & \multirow{3}{*}{ A. basal } & $\mathrm{T}$ & 1092,55 & 1088,75 & $-3,81$ & 4,05 & 0,00 & 4,05 & - & 14,96 \\
\hline & & SB & 556,45 & 561,34 & 4,89 & 0,52 & 0,00 & 0,52 & - & 29,84 \\
\hline & & $\mathrm{D}$ & 1190,19 & 1186,54 & $-3,65$ & 5,94 & 0,00 & 5,94 & - & 16,37 \\
\hline & \multirow{3}{*}{ Biomassa } & $\mathrm{T}$ & 1074,50 & 1070,93 & $-3,58$ & 3,78 & 0,00 & 3,78 & - & 16,82 \\
\hline & & SB & 509,16 & 514,01 & 4,85 & 0,43 & 0,00 & 0,43 & - & 29,81 \\
\hline & & $\mathrm{D}$ & 1178,48 & 1175,07 & $-3,41$ & 5,68 & 0,00 & 5,68 & - & 17,71 \\
\hline & \multirow{3}{*}{ Densidade } & $\mathrm{T}$ & 1709,31 & 1711,53 & 2,23 & 46,16 & 0,00 & 46,16 & - & 27,40 \\
\hline & & SB & 1726,20 & 1725,76 & $-0,44$ & 48,79 & 0,00 & 48,79 & - & 24,23 \\
\hline & & $\mathrm{D}$ & 1604,69 & 1602,11 & $-2,58$ & 30,11 & 0,00 & 30,11 & - & 20,45 \\
\hline \multirow{9}{*}{ Ombrófila } & \multirow{3}{*}{ A. basal } & $\mathrm{T}$ & 1304,94 & 1374,06 & 69,12 & 8,13 & 4,05 & 12,18 & 0,33 & 104,40 \\
\hline & & SB & 663,64 & 799,59 & 135,95 & 1,33 & 0,00 & 1,33 & - & 100,22 \\
\hline & & $\mathrm{D}$ & 1321,20 & 1363,06 & 41,86 & 6,15 & 6,52 & 12,67 & 0,51 & 122,07 \\
\hline & \multirow{3}{*}{ Biomassa } & $\mathrm{T}$ & 1626,96 & 1650,20 & 23,24 & 13,92 & 22,11 & 36,04 & 0,61 & 96,25 \\
\hline & & SB & 543,79 & 635,81 & 92,02 & 0,61 & 0,10 & 0,71 & 0,14 & 98,10 \\
\hline & & $\mathrm{D}$ & 1618,41 & 1634,53 & 16,12 & 11,30 & 25,56 & 36,86 & 0,69 & 110,34 \\
\hline & \multirow{3}{*}{ Densidade } & $\mathrm{T}$ & 1964,18 & 2143,85 & 179,66 & 254,47 & 0,00 & 254,87 & - & 126,24 \\
\hline & & SB & 1938,14 & 2107,03 & 168,89 & 220,58 & 0,00 & 220,58 & - & 119,72 \\
\hline & & $\mathrm{D}$ & 1713,43 & 1786,85 & 73,41 & 45,82 & 23,87 & 69,69 & 0,34 & 134,88 \\
\hline \multirow{9}{*}{ Restinga } & \multirow{3}{*}{ A. basal } & $\mathrm{T}$ & 965,70 & 967,41 & 1,71 & 0,23 & 2,31 & 2,54 & 0,90 & 281,15 \\
\hline & & SB & 464,72 & 476,49 & 11,77 & 0,06 & 0,31 & 0,37 & 0,85 & 222,04 \\
\hline & & $\mathrm{D}$ & 1039,56 & 1045,89 & 6,33 & 0,43 & 3,07 & 3,49 & 0,88 & 417,30 \\
\hline & \multirow{3}{*}{ Biomassa } & $\mathrm{T}$ & 842,94 & 846,94 & 4,00 & 0,18 & 1,42 & 1,60 & 0,89 & 326,67 \\
\hline & & SB & 420,91 & 430,70 & 9,79 & 0,04 & 0,27 & 0,31 & 0,86 & 231,82 \\
\hline & & $\mathrm{D}$ & 934,82 & 941,52 & 6,69 & 0,30 & 2,03 & 2,33 & 0,87 & 382,43 \\
\hline & \multirow{3}{*}{ Densidade } & $\mathrm{T}$ & 1728,16 & 1749,34 & 21,19 & 25,52 & 27,65 & 53,17 & 0,52 & 72,02 \\
\hline & & SB & 1757,13 & 1791,39 & 34,26 & 29,71 & 31,99 & 61,70 & 0,52 & 89,03 \\
\hline & & $\mathrm{D}$ & 1646,44 & 1653,82 & 7,38 & 9,67 & 27,19 & 36,87 & 0,74 & 87,37 \\
\hline
\end{tabular}

Em geral, a estrutura de continuidade espacial foi mais visível no estrato sub-bosque nas quatro formações florestais estudadas, e, consequentemente, a proporção do componente aleatório dado pelo efeito pepita relativo $\left(\operatorname{Rel}_{\tau}\right.$ ) foi menor. Contrariamente, na floresta Estacional, nos estratos total e dossel, o modelo não-espacial foi o mais plausível para as variáveis estudadas. Capretz (2004), estudando o padrão espacial das árvores em cada parcela permanente com base em seis classes de diâmetro, verificou que nas formações de Savana, Ombrófila e Restinga predominou o padrão agregado para as duas primeiras classes $(\mathrm{d}<20 \mathrm{~cm})$, e padrão aleatório para as últimas. Na floresta Estacional, o padrão espacial observado para a primeira classe foi agregado, e para as demais classes predominantemente aleatório. Comparando esses resultados, é possível sugerir que as árvores de sub-bosque têm um padrão de 
dependência espacial mais pronunciado do que o das árvores de dossel.

Na tabela 5 são apresentados o número de parâmetros e os valores do critério de informação de Akaike nos estratos total, sub-bosque e dossel para as três variáveis estudadas.

Tabela 5. Número de parâmetros (NP) e critério de informação de Akaike (AIC) para as variáveis área basal $\left(\mathrm{m}^{2} \cdot \mathrm{ha}^{-1}\right)$, biomassa $\left(\mathrm{Mg} \cdot \mathrm{ha}^{-1}\right)$ e densidade $\left(\mathrm{ha}^{-1}\right)$ nos estratos total (T), sub-bosque (SB) e dossel (D).

Table 5. Number of parameters (NP) and Akaike information criterion (AIC) for the variables basal area $\left(\mathrm{m}^{2} \cdot \mathrm{ha}^{-1}\right)$, biomass $\left(\mathrm{Mg} \cdot \mathrm{ha}^{-1}\right)$ and density $\left(\mathrm{ha}^{-1}\right)$, in stratum total (T), understory (SB) and canopy (D).

\begin{tabular}{|c|c|c|c|c|c|}
\hline Variável & Estratos & & $\operatorname{los}$ & NP & AIC \\
\hline \multirow{15}{*}{ Área basal } & \multirow{5}{*}{$\mathrm{T}$} & M1 & $\mathrm{S}=\mathrm{E}=\mathrm{O}=\mathrm{R}$ & 4 & 4405,32 \\
\hline & & M2 & $\mathrm{S} \neq \mathrm{E} \neq \mathrm{O} \neq \mathrm{R}$ & 16 & 4031,99 \\
\hline & & M3 & $(\mathrm{S}=\mathrm{R}) \neq \mathrm{E} \neq \mathrm{O}$ & 12 & 4107,53 \\
\hline & & M4 & $(S=E) \neq O \neq R$ & 12 & 4189,63 \\
\hline & & M5 & $(\mathrm{S}=\mathrm{E}=\mathrm{R}) \neq \mathrm{O}$ & 8 & 4185,91 \\
\hline & \multirow{5}{*}{ SB } & M1 & $\mathrm{S}=\mathrm{E}=\mathrm{O}=\mathrm{R}$ & 4 & 2277,48 \\
\hline & & M2 & $\mathrm{S} \neq \mathrm{E} \neq \mathrm{O} \neq \mathrm{R}$ & 16 & 2201,79 \\
\hline & & M3 & $(\mathrm{S}=\mathrm{R}) \neq \mathrm{E} \neq \mathrm{O}$ & 12 & 2207,05 \\
\hline & & M4 & $(S=E) \neq O \neq R$ & 12 & 2218,28 \\
\hline & & M5 & $(\mathrm{S}=\mathrm{E}=\mathrm{R}) \neq \mathrm{O}$ & 8 & 2224,75 \\
\hline & \multirow{5}{*}{$\mathrm{D}$} & M1 & $\mathrm{S}=\mathrm{E}=\mathrm{O}=\mathrm{R}$ & 4 & 4626,13 \\
\hline & & M2 & $\mathrm{S} \neq \mathrm{E} \neq \mathrm{O} \neq \mathrm{R}$ & 16 & 4367,44 \\
\hline & & M3 & $(S=R) \neq E \neq O$ & 12 & 4409,10 \\
\hline & & M4 & $(S=E) \neq O \neq R$ & 12 & 4494,95 \\
\hline & & M5 & $(\mathrm{S}=\mathrm{E}=\mathrm{R}) \neq \mathrm{O}$ & 8 & 4491,25 \\
\hline \multirow{15}{*}{ Biomassa } & \multirow{5}{*}{$\mathrm{T}$} & M1 & $\mathrm{S}=\mathrm{E}=\mathrm{O}=\mathrm{R}$ & 4 & 5238,60 \\
\hline & & M2 & $\mathrm{S} \neq \mathrm{E} \neq \mathrm{O} \neq \mathrm{R}$ & 16 & 4091,44 \\
\hline & & M3 & $(\mathrm{S}=\mathrm{R}) \neq \mathrm{E} \neq \mathrm{O}$ & 12 & 4165,30 \\
\hline & & M4 & $(S=E) \neq O \neq R$ & 12 & 4321,11 \\
\hline & & M5 & $(\mathrm{S}=\mathrm{E}=\mathrm{R}) \neq \mathrm{O}$ & 8 & 4327,73 \\
\hline & \multirow{5}{*}{ SB } & M1 & $\mathrm{S}=\mathrm{E}=\mathrm{O}=\mathrm{R}$ & 4 & 1928,33 \\
\hline & & M2 & $\mathrm{S} \neq \mathrm{E} \neq \mathrm{O} \neq \mathrm{R}$ & 16 & 1884,18 \\
\hline & & M3 & $(S=R) \neq E \neq O$ & 12 & 1887,26 \\
\hline & & M4 & $(S=E) \neq O \neq R$ & 12 & 1909,26 \\
\hline & & M5 & $(\mathrm{S}=\mathrm{E}=\mathrm{R}) \neq \mathrm{O}$ & 8 & 1910,26 \\
\hline & \multirow{5}{*}{$\mathrm{D}$} & M1 & $\mathrm{S}=\mathrm{E}=\mathrm{O}=\mathrm{R}$ & 4 & 5332,43 \\
\hline & & M2 & $\mathrm{S} \neq \mathrm{E} \neq \mathrm{O} \neq \mathrm{R}$ & 16 & 4508,00 \\
\hline & & M3 & $(S=R) \neq E \neq O$ & 12 & 4525,84 \\
\hline & & M4 & $(S=E) \neq O \neq R$ & 12 & 4650,29 \\
\hline & & M5 & $(\mathrm{S}=\mathrm{E}=\mathrm{R}) \neq \mathrm{O}$ & 8 & 4665,54 \\
\hline \multirow{15}{*}{ Densidade } & \multirow{5}{*}{$\mathrm{T}$} & M1 & $\mathrm{S}=\mathrm{E}=\mathrm{O}=\mathrm{R}$ & 4 & 7302,51 \\
\hline & & M2 & $\mathrm{S} \neq \mathrm{E} \neq \mathrm{O} \neq \mathrm{R}$ & 16 & 7194,13 \\
\hline & & M3 & $(\mathrm{S}=\mathrm{R}) \neq \mathrm{E} \neq \mathrm{O}$ & 12 & 7199,67 \\
\hline & & M4 & $(S=E) \neq O \neq R$ & 12 & 7214,16 \\
\hline & & M5 & $(\mathrm{S}=\mathrm{E}=\mathrm{R}) \neq \mathrm{O}$ & 8 & 7212,54 \\
\hline & \multirow{5}{*}{ SB } & M1 & $\mathrm{S}=\mathrm{E}=\mathrm{O}=\mathrm{R}$ & 4 & 7318,40 \\
\hline & & M2 & $\mathrm{S} \neq \mathrm{E} \neq \mathrm{O} \neq \mathrm{R}$ & 16 & 7242,05 \\
\hline & & M3 & $(\mathrm{S}=\mathrm{R}) \neq \mathrm{E} \neq \mathrm{O}$ & 12 & 7248,46 \\
\hline & & M4 & $(S=E) \neq O \neq R$ & 12 & 7269,36 \\
\hline & & M5 & $(\mathrm{S}=\mathrm{E}=\mathrm{R}) \neq \mathrm{O}$ & 8 & 7267,89 \\
\hline & \multirow{5}{*}{$\mathrm{D}$} & M1 & $\mathrm{S}=\mathrm{E}=\mathrm{O}=\mathrm{R}$ & 4 & 6591,78 \\
\hline & & M2 & $\mathrm{S} \neq \mathrm{E} \neq \mathrm{O} \neq \mathrm{R}$ & 16 & 6572,38 \\
\hline & & M3 & $(S=R) \neq E \neq O$ & 12 & 6567,08 \\
\hline & & M4 & $(S=E) \neq O \neq R$ & 12 & 6569,97 \\
\hline & & M5 & $(\mathrm{S}=\mathrm{E}=\mathrm{R}) \neq \mathrm{O}$ & 8 & 6563,93 \\
\hline
\end{tabular}


Pode-se observar que o modelo M1, que considerou as quatro formações florestais com a mesma estrutura espacial, teve menor plausibilidade em relação aos modelos M2 a M5. Apesar das semelhanças apresentadas entre as áreas de floresta Savana e Restinga para o padrão espacial das árvores (CAPRETZ, 2004), o regime de luz em grandes clareiras e a altura do dossel (MUNIZ, 2004), foi verificado maior plausibilidade do modelo M2 em relação ao M3. O modelo M4, que considerou a proximidade das florestas Savana e Estacional, não foi mais plausível que o modelo M2. Essa constatação pode estar relacionada à presença de condições de stress ambiental na floresta de Savana, que atua na definição da dinâmica florestal, conforme relatado por Rodrigues (1999). Apesar de algumas das formações estudadas apresentarem semelhanças entre si, quando comparadas com a estrutura florestal extremamente complexa da Floresta Ombrófila (IVANAUSKAS et al., 2000) - presença de grandes clareiras com bambus e relevo extremamente acidentado, que acabam por criar diferentes faces de exposição solar (MUNIZ, 2004) e determinam o padrão espacial das árvores (CAPRETZ, 2004) -, o modelo M2 continuou sendo o de maior plausibilidade quando comparado ao M5.

Dessa forma, o modelo M2, que considerou as formações com estruturas espaciais diferentes, mesmo penalizado por 16 parâmetros, foi o mais plausível entre os modelos avaliados (M1, M3, M4 e M5), exceto com relação ao modelo M5 na variável densidade no estrato dossel.

Uma possível explicação é que, apesar de existirem semelhanças entre as formações, os fatores climáticos e geomorfológicos são muito distintos e provavelmente acabam por ser determinantes para a estrutura e dinâmica florestal e para a estrutura de continuidade espacial.

\section{CONCLUSÕES}

Nas condições em que o presente trabalho foi desenvolvido, verificou-se que:

- As estruturas de continuidade espacial são distintas entre estratos e entre formações florestais. No entanto, foram mais visíveis no estrato sub-bosque nas variáveis área basal, biomassa e densidade nas quatro formações florestais.

- As estruturas espaciais são mais claramente detectadas nas florestas de Savana e Ombrófila, sendo que, aparentemente, se apresentam em maior escala na Savana.

- Comparando-se as estruturas espaciais das quatro formações florestais, verificou-se que o modelo mais plausível foi o que considera as estruturas como diferentes, exceto para a variável densidade no estrato dossel.

- Os métodos geoestatísticos aplicados foram capazes de captar e descrever as diferentes estruturas espaciais.

\section{AGRADECIMENTOS}

À CAPES, pela concessão de bolsa de doutorado DS-CAPES ao primeiro autor. À FAPESP, por meio do Projeto "Biota Parcela Permanentes", pela concessão dos dados para a realização deste trabalho.

\section{REFERÊNCIAS}

AKAIKE, H. A new look at statistical model identification. Transactions on Automatic Control. New York, v. 19, n. 6, p. 717-723, 1974.

BOX, G. E. P.; COX, D. R. An analysis of transformations. Journal of the Royal Statistical Society, London, v. 26, n. 2, p.211-252, 1964.

BURGER, D. M. Modelos alométricos para a estimativa da fitomassa de Mata Atlântica na Serra do Mar, SP. 112 p. Tese (Doutoradoem Ecologia) - Instituto de Biociências, Universidade de São Paulo, São Paulo, 2005

BURNHAM, K. P.; ANDERSON, D. R. Model selection and multimodel inference. 2nd ed. New York: Springer, 2002, $350 \mathrm{p}$.

CAPRETZ, R. L. Análise de padrões espaciais de árvores em quatro formações florestais do Estado 
de São Paulo, através de análises de segunda ordem, com a função K de Ripley. 93 p. Dissertação (Mestrado em Ecologia de Agroecossistemas) - Escola Superior de Agricultura "Luiz de Queiroz", Universidade de São Paulo. Piracicaba, 2004.

CHRISTENSEN, O. F.; DIGGLE, P. J.; RIBEIRO JR, P. J. Analysing positive-valued spatial data: the transformed gaussian model. In: MONESTIEZ, P.; ALLARD, D.; FROIDEVAUX, R., (Eds.). geoENV Geostatistics for Environmental Applications. Amsterdam: Kluwer, 2001, p. 287-298.

COCHRAN, W. G. Sampling techniques. 3rd Ed., New York: J.Wiley, 1977. 555 p.

DIGGLE, P. J.; TAWN, J. A.; MOYEED, R. A. Model-Based geostatistics. Applied Statistics, London, v. 47, p. 299-350, 1998.

DIGGLE, P. J.; RIBEIRO JR., P. J. Model-Based geostatistics, New York, Springer, 2007. 228 p.

GOSLEE, S. C. Behavior of vegetation sampling methods in the presence of spatial autocorrelation. Plant Ecology, Dordrecht, n. 187, p. 203-212, 2006.

IVANAUSKAS, N. M.; MONTEIRO, R.; RODRIGUES, R. R. Similaridade florística entre áreas de floresta Atlântica no estado de São Paulo. Brazilian Journal of Ecology, Rio Claro, v. 4, p. 71-81, 2000.

ISAAKS, E. H.; SRIVASTAVA, R. H. Applied Geostatisctics, New York: Oxford University Press, 1989. $561 \mathrm{p}$.

KENKEL, N. C.; JUHFISZ-NAGY, P.; PODANI, J. On sampling procedures in population and community ecology. Vegetatio, The Hague, v. 83, p. 195-207, 1989.

KOENIG, W. D. Spatial autocorrelation of ecological phenomena. Trends in Ecology \& Evolution, Amsterdam, v. 14, p. 22 - 26, 1999.

KULLBACK, S.; LEIBLER, R. A. On information and sufficiency. Annals of Mathematical Statistics, Ann Arbor, v. 22, p. 79-86, 1951.

MUNIZ, M. R. A. Estudo do regime de luz nas quatro principais formações fitogeográficas no Estado de São Paulo durante o inverno do ano de 2003. 190 p. Dissertação (Mestrado em Biologia Vegetal) - Universidade Estadual de Campinas, Campinas, 2004.

R DEVELOPMENT CORE TEAM. R: A language and environment for statistical computing. R Foundation for Statistical Computing, Vienna, Austria. Disponível em: <http://www.R-project.org>. Acesso em: 4/2/2009.

RIBEIRO JR., P. J.; DIGGLE, P. J. The geoR package functions for geostatistical data analysis. RNEWS, Vienna, v. 1, p. 15-18, 2001.

RODRIGUES, R. R. A vegetação de Piracicaba e municípios do entorno. IPEF. Circular Técnica, Piracicaba, n. 189, 20 p., 1999.

RODRIGUES, R. R. Diversidade, dinâmica e conservação em florestas do Estado de São Paulo: 40ha de parcelas permanentes. Laboratório de Ecologia e Restauração Florestal, ESALQ/USP. Piracicaba, 2002. Disponível em: <http://www.lerf.esalq.usp.br/old/parcelas/projeto.pdf>. Acesso em: 4/2/2009.

RODRIGUES, R. R. $4^{\circ}$ Relatório Temático do Projeto Parcelas Permanentes. Laboratório de Ecologia e Restauração Florestal, ESALQ/USP. Piracicaba, 2005. Disponível em: <http://www.lerf.esalq.usp.br/old/parrel2005.php>. Acesso em: 4/2/2009.

VIOLA, D. N. Deteç̧ão e modelagem de padrão espacial em dados binários e de contagem. 118 p. Tese (Doutorado) - Escola Superior de Agricultura "Luiz de Queiroz", Universidade de São Paulo, Piracicaba, 2007. 